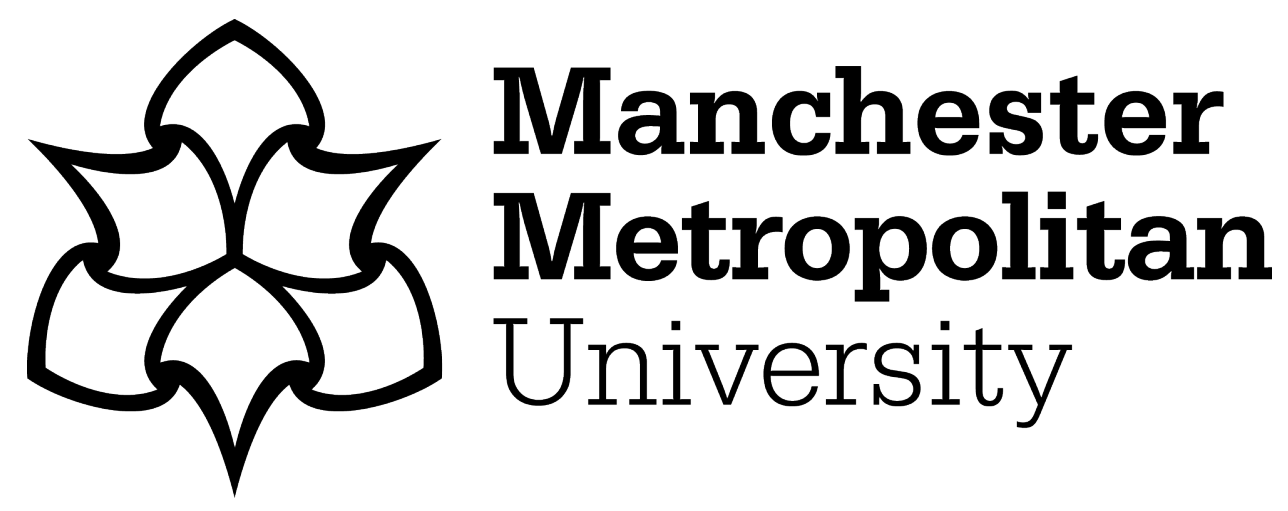

Thomas, C, Comfort, P, Dos'Santos, T ORCID logoORCID: https://orcid.org/0000-0003-2715-0116 and Jones, PA (2017) Determining Bilateral Strength Imbalances in Youth Basketball Athletes. International Journal of Sports Medicine, 38 (9). pp. 683-690. ISSN 0172-4622

Downloaded from: https://e-space.mmu.ac.uk/626033/

Version: Accepted Version

Publisher: Thieme

DOI: https://doi.org/10.1055/s-0043-112340

Please cite the published version 
See discussions, stats, and author profiles for this publication at: https://www.researchgate.net/publication/318868671

\section{Determining Bilateral Strength Imbalances in Youth Basketball Athletes}

Article in International Journal of Sports Medicine · September 2017

DOI: 10.1055/s-0043-112340

4 authors:

Christopher Thomas

56 PUBLICATIONS 609 CITATIONS

SEE PROFILE

Thomas Dos'Santos

Manchester Metropolitan University

66 PUBLICATIONS 508 CITATIONS

SEE PROFILE
Paul Comfort

University of Salford

215 PUBLICATIONS 2,264 CITATIONS

$$
\text { SEE PROFILE }
$$

Paul A Jones

University of Salford

119 PUBLICATIONS 1,351 CITATIONS

SEE PROFILE

Some of the authors of this publication are also working on these related projects:

Biomechanical Assessment of Weightlifting Derivatives View project

Exploring vertical jump performance in relation to modifiable and non-modifiable factors View project 


\title{
Determining Bilateral Strength Imbalances in Youth Basketball Athletes
}

\author{
Authors \\ Christopher Thomas, Paul Comfort, Thomas Dos'Santos, Paul A. Jones
}

\begin{abstract}
Affiliations
Directorate of Sport, Exercise and Physiotherapy, University of Salford, Manchester, United Kingdom of Great Britain and Northern Ireland
\end{abstract}

\section{Key words}

asymmetry, isokinetics, hop tests, countermovement jump, mid-thigh pull

accepted 14.05 .2017

\author{
Bibliography \\ DOI https://doi.org/10.1055/s-0043-112340 \\ Int J Sports Med 2017; 38: 683-690 \\ (c) Georg Thieme Verlag KG Stuttgart · New York \\ ISSN 0172-4622
}

\section{Correspondence}

Mr. Christopher Thomas

Directorate of Sport, Exercise and Physiotherapy

University of Salford

M6 6PU, Salford, Manchester

United Kingdom of Great Britain and Northern Ireland

Tel.: + 44/792/5871 029, Fax: + 44/792/5871029

c.thomas2@edu.salford.ac.uk

\section{ABSTRACT}

The aims of this study were: 1) to compare the strongest (D) and weakest (ND) limbs in determining muscle strength asymmetry (MSA) ratios among various muscle strength qualities and 2 ) to determine the relationships among various muscle strength qualities. 17 youth male basketball athletes (age $=17.5 \pm 0.8$ years; height $=187.1 \pm 9.4 \mathrm{~cm}$; body mass $=81.4 \pm 10.1 \mathrm{~kg}$ ) participated in this study. Knee flexor and extensor strength at $60^{\circ} \cdot \mathrm{s}^{-1}$ was assessed for both limbs with the use of isokinetic dynamometry. Other tests involved singleleg hop (SLH), countermovement jump (CMJ) and isometric mid-thigh pull (IMTP). Statistically significant differences $(p<0.05 ; d=0.40-0.98)$ were found between D and ND limbs for all strength measures. Imbalance ratio of the IMTP was significantly related to those of $\operatorname{SLH}(r=0.75, p=0.01)$ whereas no significant relationships between D:ND ratios were found from all other tests. Percentage agreements of MSA ranged from 64.7-88.2\% between muscle strength qualities. The findings provide support for the use of field tests to detect lower-limb strength imbalances. Future research should examine the effect of different asymmetry thresholds on percentage agreements to diagnose MSA and determine its relationship to athletic performance.

\section{Introduction}

Muscle strength asymmetry (MSA) refers to the relative strength differences between limbs [26]. A strength discrepancy of $\geq 10-15 \%$ between limbs is considered to represent a meaningful asymmetry [28], but it is inconclusive whether such imbalances have an impact on sports performance and the risk of injury. However, causes of MSA are multifactorial and may be due to specific unilateral sporting and or positional demands, previous injury, dominant kicking or striking limb, and limb length differences. Research attempting to classify MSA shows inconsistent results, with research classifying MSA based on limb preference [10], highest strength measure [27], and bilateral testing protocols [2]. Additionally, several methods have been proposed to calculate MSA, which subsequently elevates or reduces imbalance scores. For example, athletes who report themselves as "right limb dominant" may not truly be "right limb strength dominant" based on the test used. Additionally, MSA equations, which use the strongest (D) and weakest (ND) limbs may be misleading when analyzed longitudi- nally, if the D limb becomes the ND through injury or in response to training and competition [8]. Several tests have previously been used to determine MSA, including isokinetic dynamometry [10], single-leg hop for distance (SLH) [30], isometric mid-thigh pull (IMTP) [14] and unilateral countermovement jump (CMJ) [29], when investigating the relationship between MSA and athletic performance. Sporting movements often require athletes to produce and accept forces in multiple planes, at various speeds, all in a fluid and ever-changing environment. Therefore, it is important for researchers and practitioners to select valid and reliable tests, which accurately assess sport-specific qualities, and to enhance these qualities through various modalities to elicit adaptations in the physical qualities required for successful sport performance.

Isokinetic dynamometry testing is commonly used to assess MSA, with recent work revealing male academy rugby union athletes to produce lower values of isokinetic peak torque on the nonpreferred limb than the preferred (defined as the leg preferred to kick the ball) limb during concentric (Effect Size $(E S)=0.21-0.66$ ) 
and eccentric ( $E S=0.15-0.37)$ knee flexion and extension modes [10]. Jones and Bampouras [27] found statistically significant $(p<0.05)$ differences of $9-12 \%$ in peak torque when comparing $D$ and ND limbs during isokinetic knee extension and flexion in collegiate athletes. These findings are similar to those by Newton et al. [36] who found imbalances of $13-16 \%$ in female softball athletes. In contrast, no statistically significant differences were found between $D$ and ND limbs in professional basketball athletes [40]. However, the relationship of isokinetic strength MSA to dynamic performance MSA remains unclear. For example, isokinetic assessments have shown poor relationships with sprinting, jumping and changing direction $[9,12,31]$. Furthermore, although isokinetic assessments permit the quantification of torque, which is particularly useful for the screening of athletes, many would argue isokinetic assessments lack practical relevance and ecological validity to sport and exercise science.

Newton et al. [36] found a statistically significant $(p<0.05)$ difference in unilateral CMJ peak force (CMJ PF) between D and ND limbs, whereas Kobayashi et al. [29] found significant differences jump height $(p=0.001)$ and mean hip joint torque $(p=0.0468)$ between D and ND limbs during unilateral CMJs. In contrast, Kobayashi et al. [29] found no differences in peak and mean vertical ground reaction force. Similarly, neither study found peak force or jump height from CM] to significantly relate to any measure of isokinetic strength. However, McElveen et al. [32] found peak vertical ground reaction force of D and ND limbs to statistically correlate to unilateral CMJ height. Previous research has established unilateral CMJ to significantly relate $(r=0.64)$ to unilateral horizontal jump performance in male physical education students. However, no attempt was made to quantify the association of MSA between unilateral tests. Hart et al. [22] found lean mass asymmetry was related to reductions in kicking performance in Australian Football players, whereas Bell et al. [7] found similar results showing a negative relationship between bilateral force and power asymmetry and unloaded CMJ performance. Moreover, recent studies have shown that carry-over of force production asymmetry exists in IMTP and CMJ [2-4]. However, it should be noted that population characteristics may influence MSA carry-over characteristics; therefore; implementing only one type of MSA test and attempting to infer those findings to all strength qualities may be inappropriate. Munro and Herrington [34] found mean limb symmetry index scores (dominant limb/non-dominant limb * 100) to be $100 \%$ during SLH, indicating the there was no MSA present in male and female athletes tested. In contrast, Jones and Bampouras [27] found statistically significant $(p<0.01)$ differences between $D$ and ND limbs ( $D$ leg - ND leg/D leg x 100) in collegiate athletes. Additionally, the same authors found no relationship for D:ND ratio between SLH and isokinetic quadriceps and hamstring strength. Collectively, these studies indicate that MSA is task- and-muscle strength quality dependent, and that normative data must be generated across sports and tests to determine appropriate MSA ratios.

Asymmetrical differences have been observed between $D$ and ND limbs for IMTP peak force (IMTP PF) and time-specific force values [4], with researchers reporting larger MSA ratios in weaker athletes compared to stronger athletes, regardless of sex [4]. Furthermore, larger MSA ratios have been associated with lower jump heights in loaded and unloaded jumps [2]. However, although high- ly valuable, IMTP testing was performed bilaterally, which does not directly assess an isolated limb's force production capabilities. Recent research has found PF recorded during unilateral stance IMTP testing performed in a custom rig to reveal statistically significant $(p<0.001)$ differences in IMTP PF between D and ND limbs identifying the unilateral IMTP to be able to detect MSA, in addition to assessing one's isometric force-production capabilities. Additionally, unilateral IMTP PF has also shown a strong relationship with 505 performance $(r=-0.65)$ indicating the importance of strength of both limbs during change of direction tasks [41].

It is highly important MSA assessments that utilize similar types, magnitudes, and rates of loading specific to the mechanical variables which dictate the sport performance. Therefore, the aims of this study were: 1 ) to compare the strongest (D) and weakest (ND) limbs in determining muscle strength asymmetry (MSA) ratios among various muscle strength qualities and 2) to determine the relationships among various muscle strength qualities assessed in this study (concentric and eccentric knee extensor and flexor torque, unilateral stance IMTP peak force, vertical and horizontal jump performance). It was hypothesized that significant differences would be found between D and ND limbs for all tests. It was further hypothesized there would be no relationships between MSA ratios among isokinetic and functional field tests.

\section{Materials and Methods}

\section{Participants}

Young male basketball athletes $(n=17$; age $=17.5 \pm 0.8$ years; height $=187.1 \pm 9.4 \mathrm{~cm}$; body mass $=81.4 \pm 10.1 \mathrm{~kg}$ ) participated in this study. All athletes provided written informed consent, with consent from the parent or guardian of all athletes under the age of 18 . The study procedures were approved by the University Ethics Committee, and procedures conformed to the Declaration of Helsinki. A cross-sectional design was used to determine muscle strength imbalances of the lower limbs using isokinetic dynamometry and unilateral functional field tests such as IMTP, SLH and CMJ. For the various tests used, the performance of each leg was obtained for subsequent comparison between strength $D$ (highest value) and ND (lowest value) limbs to examine the imbalance between limbs. Correlational analyses were conducted to determine relationships between $\mathrm{D}$ and ND imbalance ratios calculated from all tests. The current investigation also adhered to the standards of the International Journal of Sports Medicine described by Harriss et al. [21].

\section{Testing procedures}

Athletes attended testing on 2 separate occasions, at the same time of day, to undertake unilateral isokinetic concentric and eccentric knee extensor and flexor strength (CON EXT, ECC EXT, CON FLEX, and ECC FLEX, respectively) and isometric mid-thigh pull testing on day 1 , with CMJ and SLH completed on day 2, separated by 48-72 h. The test order for either limb was counterbalanced for all tests to reduce order bias. All athletes rested the day before testing and were asked to attend testing in a fed and hydrated state, similar to their normal practices before training. All athletes were familiar with the testing procedures as part of their normal train- 
ing and monitoring regime. Before the start of testing, athletes performed a standardized warm-up of activation and mobilization exercises, including various bodyweight lunges and squats, followed by some low-level plyometric drills, replicating the participant's standardized warm-ups before training. Further, standardized progressive warm-ups were applied before all tests to control potential variables and improve the reliability of all tests.

\section{Isokinetic testing}

Knee flexor and extensor muscle strength was assessed at $60^{\circ} \cdot \mathrm{s}^{-1}$ in both concentric and eccentric modes using a Kin Com (Chattanooga Group, Tennessee) isokinetic dynamometer, as described in previous research [20]. The dynamometer was calibrated according to manufacturers' standardized procedures prior to data collection. Before measuring each leg, the motor axis of the dynamometer was visually aligned with the axis of the knee joint (midway between the lateral condyles of the femur and tibia). The cuff of the dynamometer lever arm was attached to the ankle, $5 \mathrm{~cm}$ proximal to the malleoli, and the moment arm recorded for gravity correction purposes. The athlete was seated and stabilized by straps so that only the knee to be tested was moving with a single degree of freedom. The back of the seat was adjusted so that hip angle was $90^{\circ}$. A specific warm-up of 8 submaximal ( $70 \%$ ) concentric knee flexion and extension movements preceded the maximal measurements. The order of tests was CON EXT, ECC EXT, ECC FLEX, CON FLEX. Peak torque was obtained from 4 maximal repetitions in each mode throughout an arc of $90^{\circ}$ (full knee extension $\left.=0^{\circ}\right)$. The resistance provided by the weight of the lower-limb was recorded at $30^{\circ}$ knee flexion for gravity correction purposes by adding (for extensors) or subtracting (for flexors) the gravity correction factor: [weight of leg] * [moment arm] * [cosine (angle of flexion)]. The highest peak flexion and extension torque of the 4 repetitions in each mode was used for further analysis, and was presented as a value relative to body mass $\left(\mathrm{Nm} \cdot \mathrm{kg}^{-1}\right)$. Data were exported in ASCII format into Microsoft Excel (version 2016, Microsoft Corp., Redmond, WA, USA) for further analysis.

\section{Isometric testing}

Unilateral stance IMTP testing was performed using a portable force platform sampling at $600 \mathrm{~Hz}$ (400 Series Performance Force Plate; Fitness Technology). The force platform was interfaced with computer software (BMS) that allows for direct measurement of forcetime characteristics and then analyzed using the BMS software. Data were filtered using a fourth order Butterworth filter with a $16 \mathrm{~Hz}$ cut-off frequency. Athletes obtained self-selected knee and hip angles $\left(\mathrm{knee}=130-150^{\circ}\right.$; hip $\left.=140-160^{\circ}\right)$ based on the reports of previous research using bilateral stance IMTP [11] to represent the posture that they would adopt during the second pull phase of a clean pull, or the start position of a dynamic mid-thigh pull. For this test, an immovable, collarless steel bar was positioned at midthigh, just below the crease of the hip, to represent the start of the second pull phase of a clean pull, using a portable IMTP rig (Fitness Technology, Adelaide, Australia). The bar height could be adjusted ( $3 \mathrm{~cm}$ increments) at various heights above the force platform to accommodate different sized athletes. Once the bar height was established, the athletes stood on the force platform, and their hands were strapped to the bar in accordance with previously established methods [39], with one foot on the force platform and the other limb unsupported and flexed $90^{\circ}$ at the knee. Each athlete was provided 2 warm-up pulls on each limb, one at $50 \%$ and one at $75 \%$ of the athletes perceived maximum effort, separated by one minute of rest. Once body position was stabilized (verified by watching the subject and force trace), the subject was given a countdown of " 3 , 2, 1, Pull." Athletes performed 3 maximal IMTP on each limb, with the instruction to pull against the bar with maximal effort as quickly as possible, and push the foot down into the force platform. Each maximal isometric trial was performed for $5 \mathrm{~s}$, and all athletes were given strong verbal encouragement during each trial. 2 min of rest was given between the maximal effort pulls. The maximum force of each limb recorded from the force-time curve during the 5-s IMTP trial was reported as the PF, and was presented as a value relative to body mass $\left(\mathrm{N} \cdot \mathrm{kg}^{-1}\right)$. The mean PF for the 3 trials was used for further analysis.

\section{Countermovement jump testing}

For the unilateral CM], athletes were instructed to perform a rapid eccentric phase, immediately followed by a rapid concentric phase with the intention to jump as high as possible. Countermovement jumps were performed with one foot on the force platform and the other limb unsupported and flexed $90^{\circ}$ at the knee, the hands on the hips, and athletes were instructed to jump as high as possible and land on 2 feet. Countermovement depth was self-selected by the athletes to maximize CMJ height and ecological validity. Athletes performed 2 practice jumps, one at $50 \%$ perceived effort and one at $75 \%$ perceived effort, separated by one minute of rest, before the maximal effort trials began. Athletes performed 3 trials on each limb, with one minute of rest between trials.

Countermovement jump data were collected using a portable force platform sampling at $1000 \mathrm{~Hz}$ (Kistler, Switzerland, Model 9286AA, SN 1209740). The force platform was interfaced with a laptop to allow for direct measurement of force-time characteristics, and recorded using Bioware software (Version 5.11; Kistler Instrument Corporation, Switzerland) and applied to a customized Microsoft Excel spreadsheet (version 2016, Microsoft Corp., Redmond, WA, USA). Prior to the onset of the countermovement, athletes remained stationary on the force platform for $2 \mathrm{~s}$ to enable an accurate measurement of bodyweight. Vertical ground reaction force data were then averaged across the first second, and the onset of the countermovement was determined when this value was reduced by 5 SDs [37]. Jump height was calculated based on the vertical velocity of the center of mass (COM) at take-off [33]. Peak force was determined from the unfiltered force-time history ${ }^{32}$ and was presented as a value relative to body mass $\left(\mathrm{N} \cdot \mathrm{kg}^{-1}\right)$. The mean PF and jump height for the 3 trials was used for further analysis.

\section{Hop testing}

The SLH was used as a measure of unilateral horizontal explosive performance. A 6- $\mathrm{m}$ long, 15-cm wide line was marked on the floor, along the middle of which was a standard tape measure, perpendicular to the starting line. The test began with athletes placing the toes on the back of the start line, before balancing on the leg to be tested, with the hands on the hips. Athletes were instructed to use a countermovement, and no restrictions were placed on body angles attained during the preparatory phase, with the instruction to 
hop as far forward as possible, taking off from one leg, before landing on the same leg. Athletes had to "stick" the landing for $2 \mathrm{~s}$, with no movement of the foot or hands touching the ground, for the trial to be counted. If the subject did not do this, the trial was discarded and another was attempted. The distance was measured to the nearest $0.01 \mathrm{~m}$ using a standard tape measure, perpendicular from the front of the start line to the posterior aspect of the back heel at the landing. Athletes performed 3 warm-up trials on each limb, followed by 3 hops for maximal horizontal distance. The mean hop distance for the 3 trials was used for further analysis ( $\triangleright$ Table $\mathbf{1}$ ).

\section{Statistical analysis}

All data are presented as means ( \pm SD). Within-session reliability of the variables was examined using the 2 way random effects model intraclass correlation coefficient (ICC), coefficient of variation (CV) and standard error of measurement (SEM). Coefficient of variation was calculated as $\left(C V \%=S D /\right.$ mean $\left.{ }^{*} 100\right)$. The SEM was calculated using the formula: (SD(pooled) * $(\sqrt{1-I C C}))[42]$. An ICC equal to or above 0.70 was considered acceptable [5]. Normality of data was assessed by Shapiro-Wilk's statistic and Q-Q plot analysis. Paired samples student t-tests were used to compare strength $D$ and ND limb for each strength measure. Asymmetry index for D and ND limbs was calculated by the formula: (dominant leg - nondominant leg/dominant leg x 100). Comparisons were made for both dominant (D) and nondominant (ND) limb variables between tasks based off the highest between-limb score. Effect sizes were also calculated according to the formula Cohen's $d=M 1-M 2 / \sigma$ pooled and interpreted as trivial $(<0.19)$, small $(0.20-0.59)$, moderate (0.60-1.19), large (1.20-1.99), and very large (2.0-4.0) [25]. Following this, athletes were classified into balanced, right dominant and left dominant for each variable based on the sample mean plus sample SD of the MSA $[1,19]$. Agreement was determined based on like-for-like classifications of asymmetry, i. e., asymmetries in CON EXT and SLH were given a value of 1, as were balanced classifications in ECC EXT and SLH. In scenarios where one test showed an asymmetry and the other balance, this was given a zero. The percentage agreement for each variable was determined based on frequency counts of like-for-like diagnoses of asymmetry (asymmetrical and balanced) divided by the number of comparisons $x 100$ to see whether categorization of MSA differed between muscle strength qualities. An agreement of $75 \%$ (i. e., agreement of 3 out of 4 athletes) was considered to identify relationships of MSA between strength qualities. Pearson's product moment correlation or nonparametric equivalent Spearman's rho was used to explore relationships among strength ratios (D:ND). Correlations were evaluated as follows: small (0.10-0.29), moderate $(0.30-$ $0.49)$, large (0.50-0.69), very large $(0.70-0.89)$, nearly perfect (0.90 to 0.99), and perfect (1.0) [25]. To avoid Type 1 error, a HolmBonferroni sequential adjustment was applied as multiple separate comparisons were completed. All statistical analyses were completed using SPSS version 23 (IBM, New York, NY, USA), and statistical significance was set at $\mathrm{p} \leq 0.05$.

\section{Results}

Statistically significant differences $(p<0.05)$ were found when comparing $D$ and ND limbs for all strength measures ( $\triangleright$ Tables 2-5),
- Table 1 Within-session reliability of muscle strength qualities. *

\begin{tabular}{|c|c|c|c|c|c|}
\hline Reliability Variable & Mean & SD & ICC & CV & SEM \\
\hline R CON EXT $\left(\mathrm{Nm} \cdot \mathrm{kg}^{-1}\right)$ & 2.52 & 0.46 & 0.99 & 18.04 & 0.05 \\
\hline L CON EXT $\left(\mathrm{Nm} \cdot \mathrm{kg}^{-1}\right)$ & 2.57 & 0.40 & 0.99 & 15.61 & 0.04 \\
\hline R ECC EXT $\left(\mathrm{Nm} \cdot \mathrm{kg}^{-1}\right)$ & 3.72 & 0.71 & 0.99 & 18.96 & 0.07 \\
\hline L ECC EXT $\left(\mathrm{Nm} \cdot \mathrm{kg}^{-1}\right)$ & 3.57 & 0.72 & 0.99 & 20.30 & 0.07 \\
\hline R CON FLEX $\left(\mathrm{Nm} \cdot \mathrm{kg}^{-1}\right)$ & 1.70 & 0.18 & 0.99 & 10.39 & 0.02 \\
\hline L CON FLEX $\left(\mathrm{Nm} \cdot \mathrm{kg}^{-1}\right)$ & 1.60 & 0.16 & 0.99 & 9.94 & 0.02 \\
\hline R ECC FLEX $\left(\mathrm{Nm} \cdot \mathrm{kg}^{-1}\right)$ & 2.04 & 0.23 & 0.99 & 11.33 & 0.02 \\
\hline L ECC FLEX $\left(\mathrm{Nm} \cdot \mathrm{kg}^{-1}\right)$ & 1.94 & 0.28 & 0.99 & 14.15 & 0.03 \\
\hline SLH R (m) & 1.69 & 0.26 & 0.97 & 2.90 & 0.04 \\
\hline $\mathrm{SLH} L(\mathrm{~m})$ & 1.68 & 0.23 & 0.94 & 3.43 & 0.06 \\
\hline CMJ JH R (m) & 0.15 & 0.04 & 0.96 & 4.95 & 0.01 \\
\hline CMJ JH L (m) & 0.15 & 0.03 & 0.89 & 5.64 & 0.01 \\
\hline IMTP PF R $\left(\mathrm{N} \cdot \mathrm{kg}^{-1}\right)$ & 26.67 & 3.38 & 0.88 & 3.92 & 1.22 \\
\hline IMTP PF L $\left(\mathrm{N} \cdot \mathrm{kg}^{-1}\right)$ & 26.83 & 3.34 & 0.86 & 4.10 & 1.30 \\
\hline CMJ PF R $\left(\mathrm{N} \cdot \mathrm{kg}^{-1}\right)$ & 20.13 & 1.65 & 0.87 & 2.68 & 0.45 \\
\hline CMJ PF L $\left(\mathrm{N} \cdot \mathrm{kg}^{-1}\right)$ & 20.54 & 1.89 & 0.94 & 2.25 & 0.49 \\
\hline \multicolumn{6}{|c|}{$\begin{array}{l}{ }^{*} \mathrm{CON}=\text { concentric; } \mathrm{ECC}=\text { eccentric; } \mathrm{EXT}=\text { extensor; } \mathrm{FLEX}=\text { flexor; } \\
\mathrm{SLH}=\text { single-leg hop; } \mathrm{CM} \text { = countermovement jump; JH= jump } \\
\text { height; IMTP = isometric mid-thigh pull; } \mathrm{PR}=\text { peak force; } \mathrm{L}=\text { left leg; } \\
\mathrm{R}=\text { right leg; } \mathrm{ICC}=\text { intraclass correlation coefficient; } \mathrm{CV}=\text { coefficient of } \\
\text { variation; SEM = standard error of measurement. }\end{array}$} \\
\hline
\end{tabular}

with small-to-moderate effects observed $(d=0.40-0.71)$ ( Tables 2-4).

Spearman's rho correlation analysis revealed a statistically significant relationship in D:ND ratio between IMTP PF and SLH distance $(r=0.75 ; p=0.01)$. However, there were no statistically significant relationships between any other tests used in the study $(p>0.05)$ ( Table 5).

- Table 6 provides frequency counts for balanced, right and left dominant classifications for each muscle strength quality based on the MSA threshold of mean \pm SD of the D:ND imbalance. $>$ Table 7 presents the agreement in diagnosis of MSA between muscle strength qualities based on mean + SD of the D:ND imbalance, ranging from $64.7 \%$ to $88.2 \%$.

\section{Discussion}

The aims of this study were: 1 ) to compare strongest (D) and weakest (ND) limbs in determining muscle strength asymmetry (MSA) ratios among various muscle strength qualities and 2 ) to determine the relationships among various muscle strength qualities (concentric and eccentric knee extensor and flexor torque, unilateral stance IMTP peak force, vertical and horizontal jump performance). In agreement with previous research $[27,36]$, this study indicated that statistically significant differences were found between $D$ and ND limbs for all test measures. The present findings also suggest a very large relationship exists between $\mathrm{N}$ :ND ratios determined via IMTP PF and SLH, whereas no significant relationship was evident between any other isokinetic or functional field tests.

The findings of this study agree with previous research that found statistically significant differences (7-13\%) between D and ND limbs for peak torque during isokinetic knee flexion and extension in athletic individuals $[27,36]$. These results are similar to those 
- Table 2 Mean \pm SD isokinetic knee extension and flexion peak torque at $60^{\circ} \cdot \mathrm{s}^{-1}$ for D and ND limbs. *

\begin{tabular}{|c|c|c|c|c|c|}
\hline & D & ND & Imbalance (\%) & p & d \\
\hline CON EXT $\left(\mathrm{Nm} \cdot \mathrm{kg}^{-1}\right)$ & $2.72 \pm 0.42$ & $2.37 \pm 0.36$ & $11.98 \pm 11.18$ & 0.001 & 0.89 \\
\hline $\operatorname{CON}$ FLEX $\left(\mathrm{Nm} \cdot \mathrm{kg}^{-1}\right)$ & $1.71 \pm 0.17$ & $1.59 \pm 0.15$ & $13.11 \pm 10.10$ & 0.004 & 0.77 \\
\hline ECC EXT $\left(\mathrm{Nm} \cdot \mathrm{kg}^{-1}\right)$ & $3.39 \pm 0.63$ & $3.39 \pm 0.71$ & $7.00 \pm 7.21$ & 0.001 & 0.75 \\
\hline $\operatorname{ECC~FLEX~}\left(\mathrm{Nm} \cdot \mathrm{kg}^{-1}\right)$ & $1.88 \pm 0.23$ & $1.88 \pm 0.23$ & $10.54 \pm 7.91$ & 0.001 & 0.98 \\
\hline
\end{tabular}

- Table 3 Mean \pm SD of horizontal and vertical jump scores for D and ND limbs. *

\begin{tabular}{|c|c|c|c|c|c|}
\hline & D & ND & Imbalance (\%) & $\mathbf{p}$ & d \\
\hline $\mathrm{SLH}(\mathrm{m})$ & $1.73 \pm 0.26$ & $1.64 \pm 0.21$ & $4.97 \pm 6.81$ & 0.027 & 0.40 \\
\hline CMJ JH (m) & $0.16 \pm 0.04$ & $0.14 \pm 0.03$ & $12.26 \pm 9.99$ & 0.001 & 0.65 \\
\hline
\end{tabular}

- Table 4 Mean \pm SD of isometric and dynamic peak force for D and ND limbs. *

\begin{tabular}{|c|c|c|c|c|c|}
\hline & D & ND & Imbalance (\%) & $\mathbf{p}$ & d \\
\hline IMTP PF $\left(\mathrm{N} \cdot \mathrm{kg}^{-1}\right)$ & $27.41 \pm 3.31$ & $26.09 \pm 3.27$ & $4.80 \pm 3.63$ & 0.001 & 0.40 \\
\hline $\mathrm{CMJ} P F\left(\mathrm{~N} \cdot \mathrm{kg}^{-1}\right)$ & $20.87 \pm 1.78$ & $19.81 \pm 1.63$ & $4.98 \pm 3.32$ & 0.001 & 0.62 \\
\hline
\end{tabular}

- Table 5 Correlation coefficients for D:ND ratio in each test. *

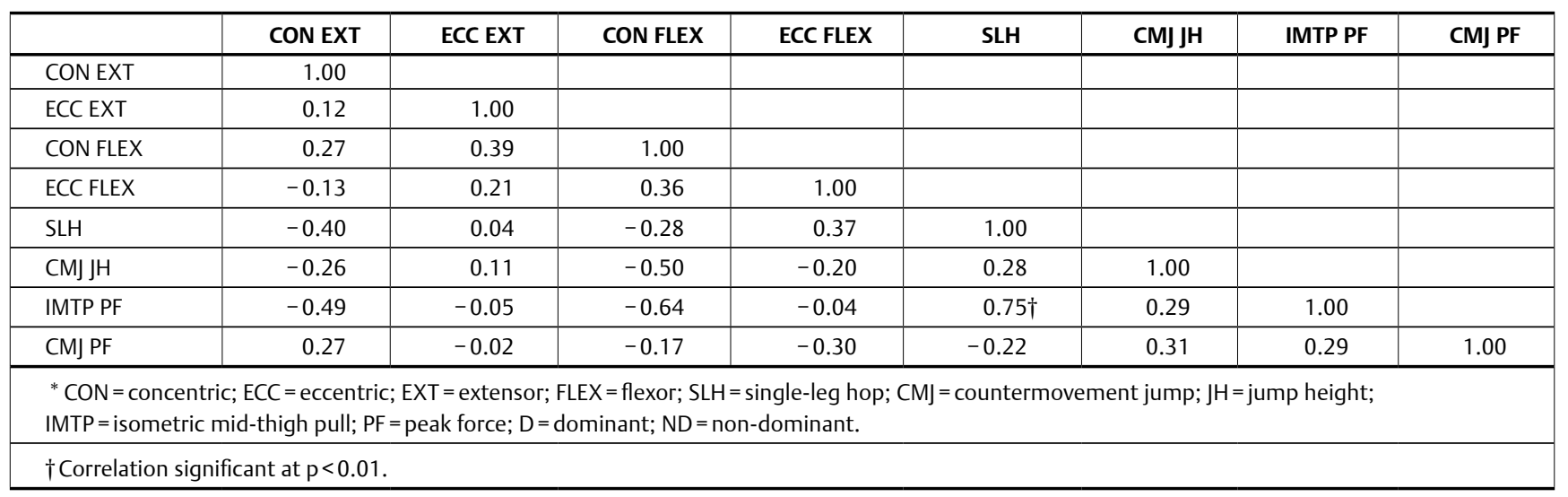

by Newton et al. [36], and Jones and Bampouras [27], who reported MSA of $6-14 \%$, but in contrast to those of Theoharopolous et al. [40], who found no statistically significant differences between D and ND limbs in professional basketball athletes. This inconsistency may be due to differences in calculating $D$ and ND limbs. In the current study and work by previous authors $[27,36]$, the $D$ limb was defined as the limb with the highest value, whereas Theoharopolous et al. [40] defined the D limb as the leg which subjects would kick a ball with, which may have influenced the findings as the $D$ (stronger) limb may not reflect the dominant action in basketball, thus affecting the MSA value. Furthermore, it is suggested that asymmetries are training- and competition-history specific $[6,8]$, so it may be that professional basketball players are equally efficient at producing and resisting force on both limbs during single-joint isokinetic assessments, compared to youth basketball athletes.

Our findings revealed percentage imbalances of $5 \%$ and $12 \%$ for SLH distance and unilateral CMJ height, respectively. These results are consistent with those that found significant differences between D and ND limbs in SLH performance in high school (2\%) [35]
- Table 6 Frequency counts of the diagnosis of muscle strength asymmetry based on mean +SD of the D:ND imbalance. *

\begin{tabular}{|c|c|c|c|}
\hline & Balanced & Right & Left \\
\hline $\operatorname{CON}$ EXT $\left(\mathrm{Nm} \cdot \mathrm{kg}^{-1}\right)$ & 14 & 1 & 2 \\
\hline ECC EXT $\left(\mathrm{Nm} \cdot \mathrm{kg}^{-1}\right)$ & 14 & 2 & 1 \\
\hline $\operatorname{CON}$ FLEX $\left(\mathrm{Nm} \cdot \mathrm{kg}^{-1}\right)$ & 14 & 3 & 0 \\
\hline $\operatorname{ECC~FLEX~}\left(\mathrm{Nm} \cdot \mathrm{kg}^{-1}\right)$ & 14 & 2 & 1 \\
\hline $\mathrm{SLH}(\mathrm{m})$ & 16 & 1 & 0 \\
\hline CMJ JH (m) & 14 & 2 & 1 \\
\hline IMTP PF $\left(\mathrm{N} \cdot \mathrm{kg}^{-1}\right)$ & 14 & 1 & 2 \\
\hline CMJ PF $\left(\mathrm{N} \cdot \mathrm{kg}^{-1}\right)$ & 15 & 0 & 2 \\
\hline \multicolumn{4}{|c|}{$\begin{array}{l}{ }^{*} \mathrm{CON}=\text { concentric; ECC = eccentric; EXT = extensor; FLEX = flexor; } \\
\text { SLH = single-leg hop; CMJ = countermovement jump; JH = jump } \\
\text { height; IMTP = isometric mid-thigh pull; PR = peak force }\end{array}$} \\
\hline
\end{tabular}

and collegiate (4\%) athletes [27]. The percentage imbalance values for unilateral CMJ height found in the current study (12\%) agree with previous research (10-12\%) [27, 29], but contradict the find- 
Table 7 The agreement in diagnosis of muscle strength asymmetry between muscle strength qualities based on mean + SD of the D:ND imbalance. *

\begin{tabular}{|c|c|c|}
\hline Comparison & $\begin{array}{c}\text { Frequency of } \\
\text { Agreement }\end{array}$ & \% Agreement \\
\hline CON EXT vs. ECC EXT & 15 & 88.2 \\
\hline CON EXT vs. CON FLEX & 14 & 82.4 \\
\hline CON EXT vs. ECC FLEX & 13 & 76.5 \\
\hline CON EXT vs. SLH & 13 & 76.5 \\
\hline CON EXT vs. CMJ JH & 12 & 70.6 \\
\hline CON EXT vs. IMTP PF & 11 & 64.7 \\
\hline CON EXT vs. CMJ PF & 11 & 64.7 \\
\hline ECC EXT vs. CON FLEX & 14 & 82.4 \\
\hline ECC EXT vs. ECC FLEX & 13 & 76.5 \\
\hline ECC EXT vs. SLH & 13 & 76.5 \\
\hline ECC EXT vs. CMJ JH & 12 & 70.6 \\
\hline ECC EXT vs. IMTP PF & 11 & 64.7 \\
\hline ECC EXT vs. CMJ PF & 11 & 64.7 \\
\hline CON FLEX vs. ECC FLEX & 13 & 76.5 \\
\hline CON FLEX vs. SLH & 13 & 76.5 \\
\hline CON FLEX vs. CM] JH & 12 & 70.6 \\
\hline CON FLEX vs. IMTP PF & 12 & 70.6 \\
\hline CON FLEX vs. CMJ PF & 11 & 64.7 \\
\hline ECC FLEX vs. SLH & 14 & 82.4 \\
\hline ECC FLEX vs. CMJ JH & 12 & 70.6 \\
\hline ECC FLEX vs. IMTP PF & 12 & 70.6 \\
\hline ECC FLEX vs. CMJ PF & 12 & 70.6 \\
\hline SLH vs. CMJ JH & 14 & 82.4 \\
\hline SLH vs. IMTP PF & 15 & 88.2 \\
\hline SLH vs. CMJ PF & 15 & 88.2 \\
\hline CMJ JH vs. IMTP PF & 12 & 70.6 \\
\hline CMJ JH vs. CMJ PF & 13 & 76.5 \\
\hline IMTP PF vs. CMJ PF & 15 & 88.2 \\
\hline \multicolumn{3}{|c|}{$\begin{array}{l}{ }^{*} \mathrm{CON}=\text { concentric; } \mathrm{ECC}=\text { eccentric; } \mathrm{EXT}=\text { extensor; } \mathrm{FLEX}=\text { flexor; } \\
\mathrm{SLH}=\text { single-leg hop; } \mathrm{CM} \text { = countermovement jump; } \mathrm{JH}=\text { jump } \\
\text { height; IMTP = isometric mid-thigh pull; } \mathrm{PF}=\text { peak force; } \mathrm{D}=\text { domi- } \\
\text { nant; } \mathrm{ND}=\text { non-dominant. }\end{array}$} \\
\hline
\end{tabular}

ings of McElveen et al. [32] (3\%). A possible explanation for this might be that basketball athletes may develop strength imbalances toward a muscle strength quality which reflects the characteristics of the sport, such as a unilateral CMJ which mimics the skill of a lay-up. In contrast, McElveen et al. [32] used physically active adults as subjects, which may explain the lack of statistical significance in their study. Another possible explanation for this is that in this experiment and in those by Jones and Bampouras [27] and Kobayashi et al. [29], the $D$ limb was defined as the limb with the highest score, whereas McElveen et al. [32] defined the D limb as the preferred limb to kick a soccer ball, which may have nullified D:ND differences. Jones and Bampouras [27] explain that athletes who are "right side dominant" may be left limb strength dominant, whereas Schlumberger et al. [38] suggest against comparing preferred and non-preferred limbs, as limb dominance may lack sufficient external validity. Likewise, de Ruiter et al. [13] found no difference in unilateral squat jump height, whereas Holsgaard-Larsen et al. [24] found no difference in unilateral CM] height, with both defining limb dominance as the preferred kicking leg. These results may be explained by the fact that the dominant limb (preferred) may not reflect the dominant action in a particular sport. In the study by de Ruiter et al. [13], athletes participated in baseball, judo, gymnastics, volleyball, cycling, and running resulting in low homogeneity of athletic backgrounds, which may be another reason for lack of any significant differences.

The current study observed statistically significant differences between $D$ and ND limbs for IMTP PF (5\%). These results are in accordance with recent work by Dos'Santos et al. [14] indicating 5-6\% imbalance between $D$ and ND limbs in professional rugby league athletes and collegiate athletes. Additionally, Bailey et al. [2] found isometric asymmetrical differences between $D$ and ND limbs, with each foot on a separate force platform during bilateral stance IMTP. However, this method is not a direct assessment of an isolated limb's isometric force production capabilities. The results of this study suggest the unilateral stance IMTP can detect lowerlimb MSA in youth basketball athletes, substantiating the hypotheses of the present study. Future work should be undertaken to investigate the magnitude of MSA in relation to dynamic performance such as vertical jumping, sprinting, and COD to determine the effectiveness of the unilateral stance IMTP in strength diagnosis and rehabilitation.

In contrast to previous research [29,32], our findings revealed statistically significant differences between $D$ and ND limbs in unilateral CMJ PF (5\%). This result may be explained by the fact that previous research was conducted on physically active individuals whereas the current investigation used basketball athletes. It may be that basketball athletes benefit from repeatedly performing single leg jump tasks, specifically taking off on one limb more frequent than the other when performing a lay-up, a drill which is commonplace in basketball training and competition. Furlong and Harrison [18] found statistically significant differences $(14 \%)$ between preferred and non-preferred limbs in plantarflexion PF during a stretchshortening cycle (SSC) task, which are greater than the level of imbalance reported here (5\%). This discrepancy could be attributed to the involvement of the SSC during the 2 tasks. In this study, athletes were instructed to perform a CMJ for maximum height with movement times ranging from 0.8 to $0.9 \mathrm{~s}$, whereas athletes in the study by Furlong and Harrison [18] were instructed to push as hard as they could while minimizing contact time. Although Furlong and Harrison [18] did not report contact times, the task of interest has previously reported contact times of $<0.25 \mathrm{~s}$ [17], indicating fast SSC tasks may induce greater MSA ratios than slow SSC tasks. This theory is supported by Flanagan and Harrison [16], who found statistically significant differences $(\sim 20 \%)$ between $D$ and ND between drop jump and rebound jump protocols in physically active subjects.

The present study found IMTP PF had large, statistically significant relationships with $\operatorname{SLH}(r=0.75, p=0.01)$ ratios, whereas no statistically significant relationships between D:ND ratios were found from all other tests. Although these tests measure different muscle qualities, the relationships may be partly explained by SLH being heavily dependent on an athlete's ability to rapidly apply and yield unilateral force over short time intervals. Additionally, it may be the case that functional field tests such as the unilateral IMTP and SLH may be able to detect lower-limb MSA imbalances (88.2\% 
agreement) within the current participant population. Further research will need to be done to determine whether this remains in other sporting populations. When examining the agreement between tasks, 14 athletes were categorized as balanced, one participant was right limb dominant on both tasks, and 2 athletes were categorized as left limb dominant IMTP, but balanced during SLH. Despite this study revealing a statistically significant relationship between the D:ND ratios of these tests, these findings suggest that although agreement of MSA diagnosis is high, this may have limited impact in the strength and conditioning and clinical environment.

With the exception of IMTP and SLH, this study has been unable to demonstrate statistically significant relationships between D:ND ratios in isokinetic and functional field tests. This suggests that although functional field tests can detect MSA, their magnitude are test and muscle strength quality specific. This is likely due to the field tests involving multiple joints and antagonist co-contractions. Similarly, isokinetic assessments are limited to isolated muscle groups with a constant angular velocity, thus maybe lacking ecological validity and practical relevance. This outcome is contrary to previous studies which have suggested that carry-over of force production asymmetry exists in IMTP and CM] $[3,4]$. However, it should be noted that MSA carry-over may be population-specific. It seems possible that basketball athletes may develop strength imbalances toward a muscle strength quality which reflects the characteristics of the sport, as observed in previous studies [2-4]. Furthermore, Hewit et al. [23] found that asymmetry in one test does not predict asymmetry in another; therefore, when screening athletes, the choice of tests used and variables measured should be specific to the requirements of the sport and or positions.

Previous research has revealed inconclusive evidence as to whether MSA is associated with performance differences between limbs [30,31]. This is due to a lack of agreement on the best approach to diagnose MSA. Previous suggestions for detecting MSA have suggested $>10-15 \%$ between limbs [28]. However, MSA is dependent on the mode of assessment and muscle strength quality under investigation [8]. Aldukail et al. [1] and Graham-Smith et al. [19] have suggested the use of mean + SD of imbalance in order to determine cut-off criteria for detecting MSA, revealing differences in cut-off percentages for isokinetic, hop and vertical jump tests [1]. Lockie et al. [30] have also used the mean + (0.2 * SD) asymmetry score to evaluate whether MSA impacts change of direction performance, but found no relationship. However, the authors only investigated athletes of greater (8-13 subjects) or lower asymmetry (17-22 subjects) instead of categorizing athletes as balanced, right and left strength dominant. This study has demonstrated lower agreement in diagnosis of MSA between isokinetic dynamometry and functional field tests (64.7-76.5\%), compared to higher agreement between individual functional field tests (70.6-88.2\%), suggesting MSA's field tasks are reflected in 3-4 out of 5 subjects in another task. These findings may be somewhat limited by the cut-off frequency used in the current study. For example, if the current study used the mean $+(0.2 *$ SD) asymmetry score to detect MSA [30] this may have produced different interrelationships and levels of agreement between performance tests. Future research may benefit from examining the effect of different asymmetry thresholds on percentage agreements of like-for-like identifications and interpretations of MSA to establish the most suitable asymmetry threshold for future practice [15].

A further limitation of the study is that the use of the mean + SD imbalance criteria is based on "normative" data from the specific population under investigation, which may not be "ideal”. Future research is required to gather normative data on a range of athletes from different sports in order to establish criteria to diagnose MSA for specific muscle strength qualities. Further studies that take this criterion into account will need to be undertaken to investigate the implications of MSA on performance and risk of injury.

\section{Practical applications}

This study has shown that functional field tests are able to detect lower-limb MSA between D and ND limbs in youth male basketball athletes. These findings suggest that researchers and practitioners should consider using functional field tests to determine magnitudes of MSA, so subsequent training programs may be implemented accordingly. Additionally, imbalance ratios determined from IMTP were strongly related to those from the SLH testing. Associations based on the like-for-like agreement in diagnosis of MSA was a novel approach; however, its implications for diagnosing MSA and overall relationship to athletic performance requires further examination. The findings of this study could be used to report potential cut-off imbalance ratios for youth male basketball athletes. Although the current study is based on a small sample of athletes, the findings suggest researchers and practitioners should consider assessing MSA by due consideration of the muscle strength qualities that reflect the demands of the sport.

\section{Conclusions}

The present study found statistically significant differences between D and ND limbs for all methods of assessment. Future research should focus on determining whether the magnitude of MSA, using the reported cut-off imbalances, affects injury and or performance.

\section{Acknowledgement}

The authors would like to thank Dr Philip Graham-Smith for the permission and use of his analsysis spreadsheet. No grant funding was received to support this research.

\section{Conflict of Interest}

The Authors have no conflict of interest to declare.

\section{References}

[1] Aldukhail A, Jones P, Gillard H, Graham-Smith P. Clinical diagnosis of strength and power asymmetry. Biol Sport 2013; 15: 33-38

[2] Bailey C, Sato K, Alexander R, Chiang C-YH, Stone M. Isometric force production symmetry and jumping performance in collegiate athletes. J Trainol 2013; 2: 1-5 
[3] Bailey CA, Sato K, Burnett A, Stone MH. Carry-over of force production symmetry in athletes of differing strength levels. J Strength Cond Res 2015; 29: 3188-3196

[4] Bailey CA, Sato K, Burnett A, Stone MH. Force-production asymmetry in male and female athletes of differing strength levels. Int J Sports Physiol Perform 2015; 10: 504-508

[5] Baumgartner TA, Chung $\mathrm{H}$. Confidence limits for intraclass reliability coefficients. Meas Phys Educ Exerc Sci 2001; 5: 179-188

[6] Bazyler CD, Bailey CA, Chiang C-Y, Sato K, Stone MH. The effects of strength training on isometric force production symmetry in recreationally trained males. J Trainol 2014; 3: 6-10

[7] Bell D, Sanfilippo J, Binkley N. Lean mass asymmetry influences force and power asymmetry during jumping in collegiate athletes. J Strength Cond Res 2014; 28: 884-891

[8] Bishop C, Read P, Chavda S, Turner A. Asymmetries of the lower limb: The calculation conundrum in strength training and conditioning. Strength Cond J 2016; 38: 27-32

[9] Blackburn J, Morrissey M. The relationship between open and closed kinetic chain strength of the lower limb and jumping performance. J Orthop Sports Phys Ther 1998; 27: 430-435

[10] Brown SR, Brughelli M, Bridgeman LA. Profiling isokinetic strength by leg preference and position in rugby union athletes. Int J Sports Physiol Perform 2016; 11: 500-507

[11] Comfort P, Jones PA, McMahon JJ, Newton R. Effect of knee and trunk angle on kinetic variables during the isometric midthigh pull: Test-retest reliability. Int J Sports Physiol Perform 2015; 10: 58-63

[12] Cronin JB, Hansen KT. Strength and power predictors of sports speed. J Strength Cond Res 2005; 19: 349-357

[13] de Ruiter C], de Korte A, Schreven S, De Haan A. Leg dominancy in relation to fast isometric torque production and squat jump height. Eur J Appl Physiol 2010; 108: 247-255

[14] Dos'Santos T, Thomas C, Jones P, Comfort P. Assessing muscle strength asymmetry via a unilateral stance isometric mid-thigh pull. Int J Sports Physiol Perform 2016

[15] Exell TA, Gittoes M], Irwin G, Kerwin DG. Gait asymmetry: Composite scores for mechanical analyses of sprint running. J Biomech 2012; 45: 1108-1111

[16] Flanagan EP, Harrison AJ. Muscle dynamics differences between legs in healthy adults. J Strength Cond Res 2007; 21: 67-72

[17] Furlong L-AM, Harrison AJ. Reliability and consistency of plantarflexor stretch-shortening cycle function using an adapted force sledge apparatus. Physiol Meas 2013; 34: 437

[18] Furlong L-AM, Harrison AJ. Differences in plantarflexor function during a stretch-shortening cycle task due to limb preference. Laterality: Asymmetries of Body, Brain Cognit 2015; 20: 128-140

[19] Graham-Smith P, Al-Dukhail A, Jones P. Agreement between attributes associated with bilateral jump asymmetry. In, ISBS-Conference Proceedings Archive; 2015;

[20] Graham-Smith P, Jones PA, Comfort P. Assessment of knee flexor and extensor muscle balance. Int J Athl Ther Train 2013; 18: 1-5

[21] Harriss D, Atkinson G. Ethical standards in sport and exercise science research: 2016 update. Int J Sports Med 2015; 36: 1121-1124

[22] Hart NH, Nimphius S, Spiteri T, Newton RU. Leg strength and lean mass symmetry influences kicking performance in Australian Football. J Sports Sci Med 2014; 13: 157

[23] Hewit JK, Cronin JB, Hume PA. Asymmetry in multi-directional jumping tasks. Phys Ther Sport 2012; 13: 238-242

[24] Holsgaard-Larsen A, Jensen C, Mortensen NHM, Aagaard P. Concurrent assessments of lower limb loading patterns, mechanical muscle strength and functional performance in ACL-patients - A cross-sectional study. Knee 2014; 21: 66-73
[25] Hopkins W, Marshall S, Batterham A, Hanin J. Progressive statistics for studies in sports medicine and exercise science. Med Sci Sports Exerc 2009; 41: 3

[26] Impellizzeri FM, Rampinini E, Maffiuletti N, Marcora SM. A vertical jump force test for assessing bilateral strength asymmetry in athletes. Med Sci Sports Exerc 2007; 39: 2044-2050

[27] Jones PA, Bampouras TM. A Comparison of isokinetic and functional methods of assessing bilateral strength imbalance. J Strength Cond Res 2010; 24: 1553-1558

[28] Kannus P. Isokinetic evaluation of muscular performance: implications for muscle testing and rehabilitation. Int J Sports Med 1994; 15: S11-S18

[29] Kobayashi Y, Kubo J, Matsubayashi T, Matsuo A, Kobayashi K, Ishii N. Relationship between bilateral differences in single-leg jumps and asymmetry in isokinetic knee strength. J Appl Biomech 2013; 29: 61-67

[30] Lockie RG, Callaghan S], Berry SP, Cooke ERA, Jordan CA, Luczo TM, Jeffriess MD. Relationship between unilateral jumping ability and asymmetry on multidirectional speed in team-sport athletes. J Strength Cond Res 2014; 28: 3557-3566

[31] Lockie RG, Schultz AB, Jeffriess MD, Callaghan SJ. The relationship between bilateral differences of knee flexor and extensor isokinetic strength and multi-directional speed. Isokinet Exerc Sci 2012; 20: 211-219

[32] McElveen MT, Riemann BL, Davies G]. Bilateral comparison of propulsion mechanics during single-leg vertical jumping. J Strength Cond Res 2010; 24: 375-381

[33] Moir GL. Three different methods of calculating vertical jump height from force platform data in men and women. Meas Phys Educ Exerc Sci 2008; 12: 207-218

[34] Munro AG, Herrington LC. Between-session reliability of four hop tests and the agility T-Test. J Strength Cond Res 2011; 25: 1470-1477

[35] Myers BA, Jenkins WL, Killian C, Rundquist P. Normative data for hop tests in high school and collegiate basketball and soccer players. Int J Sports Phys Ther 2014; 9: 596-603

[36] Newton RU, Gerber A, Nimphius S, Shim JK, Doan BK, Robertson M, Pearson DR, Craig BW, Hakinnen K, Kraemer W]. Determination of functional strength imbalance of the lower extremities. J Strength Cond Res 2006; 20: 971-977

[37] Owen NJ, Watkins J, Kilduff LP, Bevan HR, Bennett MA. Development of a criterion method to determine peak mechanical power output in a countermovement jump. J Strength Cond Res 2014; 28: 1552-1558

[38] Schlumberger A, Laube W, Bruhn S, Herbeck B, Dahlinger M, Fenkart $\mathrm{G}$, Schmidtbleicher D, Mayer F. Muscle imbalances-fact or fiction? Isokinet Exerc Sci 2006; 14: 3-11

[39] Stone MH, Sands WA, Pierce KC, Carlock J, Cardinale M, Newton RU. Relationship of maximum strength to weightlifting performance. Med Sci Sports Exerc 2005; 37: 1037-1043

[40] Theoharopolous A, Tsitskaris G, Tsaklis P. Knee Strength of Professional Basketball Players. J Strength Cond Res 2000; 14: 457-463

[41] Thomas C, Dos'Santos T, Comfort PA, Jones P. Relationship between isometric strength, sprint, and change of direction speed in male academy cricketers. J Trainol 2016; 5: 18-23

[42] Thomas JR, Nelson JK, Silverman SJ. Research methods in physical activity. Champaign, IL: Human Kinetics; 2010 